\title{
Almost nilpotency of an associative algebra with an almost nilpotent fixed-point subalgebra
}

\author{
N. Yu. Makarenko* \\ Sobolev Institute of Mathematics, Novosibirsk, 630 090, Russia \\ natalia_makarenko@yahoo.fr
}

\begin{abstract}
Let $A$ be an associative algebra of arbitrary dimension over a field $F$ and $G$ a finite group of automorphisms of $A$ of order $n$, prime to the characteristic of $F$. Denote by $A^{G}=\left\{a \in A \mid a^{g}=a\right.$ for all $\left.g \in G\right\}$ the fixed-point subalgebra. By the classical Isaacs-Bergman theorem, if $A^{G}$ is nilpotent of index $d$, i.e. $\left(A^{G}\right)^{d}=0$, then $A$ is also nilpotent and its nilpotency index is bounded by a function depending only on $n$ and $d$. We prove, under additional assumption of solubility of $G$, that if $A^{G}$ contains a two-sided nilpotent ideal $I \triangleleft A^{G}$ of nilpotency index $d$ and of finite codimension $m$, then $A$ contains a nilpotent two-sided ideal $H \triangleleft A$ of nilpotency index bounded by a function of $n$ and $d$ and of finite codimension bounded by a function of $m, n$ and $d$. An even stronger result is provided for graded associative algebras: if $G$ is a finite (not necessarily soluble) group of order $n$ and $A=\bigoplus_{g \in G} A_{g}$ is a $G$-graded associative algebra over a field $F$, i.e. $A_{g} A_{h} \subset A_{g h}$, such that the identity component $A_{e}$ has a two-sided nilpotent ideal $I_{e} \triangleleft A^{G}$ of index $d$ and of finite codimension $m$ in $A_{e}$, then $A$ has a homogeneous nilpotent two-sided ideal $H \triangleleft A$ of index bounded by a function on $n$ and $d$ and of finite codimension bounded by a function on $n, d$ and $m$.
\end{abstract}

Keywords. associative algebra, actions of finite groups of automorphisms, finite grading, graded associative algebra, fixed-point subalgebra, almost nilpotency.

\section{Introduction}

By the classical Isaacs-Bergman theorem [1, if an associative algebra $A$ over a field $F$ admits a finite group of automorphisms $G$ of order $n$, prime to the characteristic of $F$, and the fixed-point subalgebra $A^{G}=\left\{a \in A \mid a^{g}=a\right.$ for all $\left.g \in G\right\}$ is nilpotent of index $d$, i.e. $\left(A^{G}\right)^{d}=0$, then $A$ is nilpotent of index bounded by a function of $n$ and $d$. Starting from this work, a great number of paper was devoted to study of properties of algebras

*The research is supported by RSF (project N 14-21-00065)

Mathematics Subject Classification (2010): Primary 16W20, 16W22, 16W50 
(or rings) subject to corresponding properties of fixed-point algebra under finite group actions. In this paper we prove, under the additional assumption of the solubility of the automorphism group, that the "almost nilpotency" of the fixed-point subalgebra implies the "almost nilpotency" of the algebra itself. Namely, the following theorem holds.

Theorem 1.1. Let $A$ be an associative algebra of arbitrary (possibly infinite) dimension over a field $F$ acted on by a finite soluble group $G$ of order $n$. Suppose that the characteristic of $F$ does not divide $n$. If the fixed-point subalgebra $A^{G}$ has a nilpotent two-sided ideal $I \triangleleft A^{G}$ of nilpotency index $d$ and of finite codimension $m$ in $A^{G}$, then $A$ has a nilpotent two-sided ideal $H \triangleleft A$ of nilpotency index bounded by a function of $n$ and $d$ and of finite codimension bounded by a function of $m, n$ and $d$.

The restrictions on the order of the automorphism group are unavoidable. There are examples showing that no results of this kind are possible either for infinite automorphism groups or for algebras with $n$-torsion.

Theorem 1.1 follows by induction on the order of $G$ from the Bergman-Isaacs theorem and the following statement on graded associative algebras, in which we do not suppose either $G$ to be soluble or the order of $G$ to be prime to the characteristic of the field.

Theorem 1.2. Let $G$ be a finite group of order $n$ and let $A=\bigoplus_{g \in G} A_{g}$ be a G-graded associative algebra over a field $F$, i.e. $A_{g} A_{h} \subset A_{g h}$. If the identity component $A_{e}$ has a nilpotent two-sided ideal $I_{e} \triangleleft A_{e}$ of nilpotency index $d$ and of finite codimension $m$ in $A_{e}$, then $A$ has a homogeneous nilpotent two-sided ideal $H \triangleright A$ of nilpotency index bounded by a function on $n$ and $d$ and of finite codimension bounded by a function on $n, d$ and $m$.

The proof of Theorem 1.2 is based on the method of generalized centralizers, originally created by Khukhro in [3] for nilpotent groups and Lie algebras with an almost regular automorphism of prime order. In [4, 5] the approach was significantly revised and new techniques were introduced to study a more complicated case of an almost regular automorphism of arbitrary (not necessarily prime) finite order. In particular, it was proved that if a Lie algebra $L$ admits an automorphism $\varphi$ of finite order $n$ with finite-dimensional fixed-point subalgebra of dimension $\operatorname{dim} \mathrm{C}_{\mathrm{L}}(\varphi)=\mathrm{m}$, then $L$ has a soluble ideal of derived length bounded by a function of $n$ whose codimension is bounded by a function of $m$ and $n$. The fundamental combinatorial nature of the construction in [5] makes possible to apply it to a wide range of situations. For example, the approach was used to study Lie type algebras (a large class of algebras which includes associative, Lie algebras, color Lie superalgebras) with an almost regular automorphism of finite order in [6].

In the proof of Theorem [1.2 we use virtually the same construction as in [5]. But the strong condition of associativity simplifies the reasoning and allows to provide much stronger results than in the case of Lie algebras. In particular, we do not need to suppose that the automorphism group is cyclic.

We give some definitions and auxiliary lemmas in $\S 2$. In $\S 3$ we prove Theorem 1.2 , For this, we set $N=d^{2}+1$ and for each $g \in G \backslash e$ we construct by induction generalized centralizers $A_{g}(i)$ of levels $i=1,2, \ldots N$, which are some subspaces of the homogeneous components $A_{g}$. Then we demonstrate that the ideal generated by all the $A_{g}(N), g \in G \backslash e$ 
is the required one. In $\S 4$ by induction on the order of $G$ we derive the Theorem 1.1 from Theorem 1.2 and the Bergman-Isaacs Theorem.

Throughout the paper we will say that a number is " $(a, b, \ldots)$ - bounded" if it is "bounded above by some function depending only on $a, b, \ldots$ ".

\section{Preliminaries}

If $G$ is a group of automorphisms of $A$, then $A^{G}=\left\{a \in A \mid a^{g}=a\right.$ for all $\left.g \in G\right\}$ will denote the fixed-point subalgebra. The two-sided ideal $H$ of $A$ is denoted by $H \triangleleft A$. If $I$ and $J$ are subspaces of $A, I J$ will denote the subspace spanned by all products $a b$ with $a \in I$ and $b \in J$, and $I^{d}$ will denote the $d$-fold product $\underbrace{I \ldots I}_{d}$. We say that an algebra is nilpotent of (nilpotency) index $d$ if the product of any $d$ elements of the $\operatorname{ring} A$ equals zero, i.e. $A^{d}=0$. The subalgebra generated by subspaces $B_{1}, B_{2}, \ldots, B_{s}$ is denoted by $\left\langle B_{1}, B_{2}, \ldots, B_{s}\right\rangle$, and the two-sided ideal generated by $B_{1}, B_{2}, \ldots, B_{s}$ is denoted by id $\left\langle B_{1}, B_{2}, \ldots, B_{s}\right\rangle$. If $H$ is an algebra, then $H^{\#}$ will denote the algebra obtained by adjoining 1 to $H$. The (two-sided) ideal of $H$ generated by a subspace $I$ is sometimes written as $H^{\#} I H^{\#}$.

We now state some facts needed in the proof.

Lemma 2.1. (Bergman-Isaacs Theorem [1]). Let $G$ be a finite group of automorphisms of an associative ring (algebra) $R$ of order $n$. If $R$ has no $n$-torsion and $R^{G}$ is nilpotent of index $d$ then $R$ is nilpotent of index at most $h^{d}$, where $h=1+\prod_{i=0}^{n}\left(C_{n}^{i}+1\right)$.

The following two lemmas are known. We give their proofs for the convenience of readers.

Lemma 2.2. (Bergman-Isaacs [1, lemma 1.1]). Let $G$ be a finite group of order $n$ and let $A=\bigoplus_{g \in G} A_{g}$ be a G-graded associative algebra over a field $F$, i.e. $A_{g} A_{h} \subset A_{g h}$. If the identity component $A_{e}$ is nilpotent of index $d$, then $A$ is nilpotent of index at most nd.

Proof. It suffices to prove that a product $a_{g_{1}} a_{g_{2}} \ldots a_{g_{n d}}$ in homogeneous elements $a_{g_{i}} \in A_{g_{i}}$, $i=1, \ldots, n d$ of length $n d$ is trivial. We consider $n d+1$ products $h_{0}=e, h_{1}=g_{1}$, $h_{i}=g_{1} \ldots g_{i}, i=1, \ldots, n d$. Since the order of $G$ is $n$, some $d+1$ elements must be equal. If $h_{i}=h_{j}$ with $i<j$, then $g_{i+1} \ldots g_{j}=e$. We obtain that $a_{g_{1}} a_{g_{2}} \ldots a_{g_{n d}}$ can be represented as $P_{1} Q_{1} Q_{2} \ldots Q_{d} P_{2}$, where $P_{1}$ and $P_{2}$ are (possibly empty) products in homogeneous $a_{g_{i}}$, and each $Q_{i}$ is a non-empty product of the form $Q_{i}=a_{g_{i+1}} \ldots a_{g_{j}}$ with $g_{i+1} \ldots g_{j}=e$. It follows that $Q_{i} \in A_{e}$ for all $i=1, \ldots, d$. Since $\left(A_{e}\right)^{d}=0$, we have that $Q_{1} Q_{2} \ldots Q_{d}=0$, and therefore $a_{g_{1}} a_{g_{2}} \ldots a_{g_{n d}}=0$.

Lemma 2.3 ([2, Lemma 1.3.7 ]). Let $A$ be an associative algebra over a field $F$ acted on by a finite group $G$ of order $n$. Suppose that the characteristic of $F$ does not divide $n$. If the subalgebra of invariants $A^{G}$ contains a nilpotent ideal $I \triangleleft A^{G}$ of nilpotency index $d$, then $A$ contains a $G$-invariant ideal $J \geqslant I$ of $(n, d)$-bounded nilpotency index. 
Proof. Consider the right-sided ideal $B=I A^{\#}$ generated by $I$ (recall that $A^{\#}$ is the algebra obtained from $A$ by joining the unit). Let $b \in B^{G}$ be an element of $B$ fixed by $G$, i. e. $b^{g}=b$ for all $g \in G$. There exist elements $s \in B, i_{m} \in I, a_{m} \in A^{\#}$ such that $b=n s=n \sum_{m} i_{m} a_{m}$. Since $\sum_{g \in G} a_{m}^{g} \in A^{G}$ and $I$ is an ideal in $A^{G}$, we have

$$
b=n s=\sum_{g \in G} s^{g}=\sum_{m} i_{m} \sum_{g \in G} a_{m}^{g} \in I,
$$

i. e. $B^{G} \leqslant I$ and $B^{G}$ is nilpotent of index $\leqslant d$. Applying Bergman-Isaacs Theorem to the algebra $B$ we obtain that $B$ is nilpotent of index at most $h^{d}$, where $h=1+\prod_{i=0}^{n}\left(C_{n}^{i}+1\right)$. Finally, two-sided $G$-invariant ideal $J \geqslant I$ generated by $B$ is also nilpotent of index at most $h^{d}:\left(A^{\#} I A^{\#}\right)^{h^{d}}=A^{\#}\left(I A^{\#}\right)^{h^{d}}=0$.

\section{Proof of Theorem 1.2}

Let $G$ be an arbitrary finite group of order $n$ and $A=\bigoplus_{g \in G} A_{g}$ be a $G$-graded associative algebra over a field $F$, i.e. $A_{g} A_{h} \subset A_{g h}$. Suppose that the identity component $A_{e}$ has a nilpotent ideal $I_{e}$ of nilpotency index $d$ and $\operatorname{dim} A_{e} / I_{e}=m$.

Index Convention. In what follows, unless otherwise stated, a small letter with an index $g$ will denote an element of the homogeneous component $A_{g}$. The index only indicates which component this element belongs to: $x_{g} \in A_{g}$. To lighten the notation, we shall not be using numbering indices for elements of the $A_{g}$, so that different elements can be denoted by the same symbol. For example, $x_{g}$ and $x_{g}$ can be different elements of $A_{g}$.

Construction of generalized centralizers and representatives. We fix $N=$ $d^{2}+3$. In each homogeneous component $A_{g}, g \in G \backslash\{e\}$ we construct by induction a descending chain of subspaces:

$$
A_{g}=A_{g}(0) \geqslant A_{g}(1) \geqslant \cdots \geqslant A_{g}(N) .
$$

The subspaces $A_{g}(s)$ are called generalized centralizers of level $s$. Simultaneously we fixe some homogeneous elements in $A_{g}(s), s=0, \ldots, N$ which are referred to as representatives of level $s$. The total number of representatives will be $(n, d, m)$-bounded.

Definition. For a monomial $a_{g_{1}} a_{g_{2}} \ldots a_{g_{k}}$, where $a_{g_{i}} \in A_{g_{i}}$, the record $\left(*_{g_{1}} *_{g_{2}} \cdots *_{g_{k}}\right)$ is called the pattern of the monomial. The length of a pattern is the degree of the monomial. The monomial is said to be the value of its pattern on the given elements.

For example, $a_{g} a_{g} a_{v}$ and $b_{g} c_{g} b_{v}$ are values of the same pattern $\left(*_{g} *_{g} *_{v}\right)$. (Under the Index Convention the elements $a_{g}$ in the first product can be different.)

Definition. Let $g \in G \backslash\{e\}$. For every ordered tuple of elements $\vec{x}=\left(x_{g_{1}}, \ldots, x_{g_{k}}\right)$, $x_{g_{s}} \in A_{g_{s}}$, such that $g_{1} g_{2} \ldots g_{l-1} g g_{l} \ldots g_{k}=e$ for some $l \in\{1, \ldots k+1\}$ we define the mappings:

$$
\vartheta_{\vec{x}, l}: A_{g} \rightarrow A_{e} / I_{e} ;
$$




$$
\vartheta_{\vec{x}, l}: y_{g} \rightarrow x_{g_{1}} x_{g_{2}} \ldots x_{g_{l-1}} y_{g} x_{g_{l}} \ldots x_{g_{k}} I_{e}
$$

where $I_{e}$ is the nilpotent ideal of $A_{e}$ of nilpotency index $d$ and of codimension $m$ in $A_{e}$. We use index $l$ to distinguish eventual cases of $g_{1} g_{2} \ldots g_{k-1} g g_{k} \ldots g_{k}=e$ and $g_{1} g_{2} \ldots g_{l-1} g g_{l} \ldots g_{k}=e$ with $k \neq l$ which lead to different mappings.

By linearity, the mapping $\vartheta_{\vec{x}, l}$ is a homomorphism of the subspace $A_{g}$ into factor-space $A_{e} / I_{e}$. Since $\operatorname{dim} A_{e} / I_{e} \leqslant m$, we have $\operatorname{dim} A_{g} / \operatorname{Ker} \vartheta_{\vec{x}, l} \leqslant m$.

Definition of level 0. We set $A_{g}(0)=A_{g}$ for all $g \in G \backslash\{e\}$. To construct representatives of level 0 we fix some elements $x_{e} \in A_{e}$ whose images form a basis of $A_{e} / I_{e}$. These elements are called representatives of level 0 and are denoted by $x_{e}(0)$ (under the Index Convention). In addition we consider a pattern $\mathbf{P}=\left(*_{g} *_{g^{-1}}\right)$ of length 2 with $g \in G \backslash\{e\}$. The dimension of the subspace of the factor-space $A_{e} / I_{e}$ spanned by all images of values of $\mathbf{P}$ on homogeneous elements of $A_{g}, A_{g^{-1}}$ is at most $m$ by hypothesis. Hence we can choose at most $m$ products $c=x_{g} x_{g^{-1}} \in A_{e}$ whose images form a basis of this subspace. The elements $x_{g}, x_{g^{-1}}$ involved in these representations of the elements $c$ are also called representatives of level 0 and are denoted by $x_{g}(0), x_{g^{-1}}(0)$ (under the Index Convention). The same is done for every pattern $\mathbf{P}$ of the form $\left(*_{g} *_{g^{-1}}\right), g \in G \backslash\{e\}$.

Since $\operatorname{dim} A_{e} / I_{e} \leqslant m$ and the total number of patterns $\mathbf{P}$ is $n-1$, the number of representatives of level 0 is at most $2(n-1) m+m$.

Definition of level 1. Let $W_{1}=2 d^{3}(n-1)+2$. For each $g \in G \backslash\{e\}$ we set

$$
A_{g}(1)=\bigcap_{\vec{z}} \bigcap_{l} \operatorname{Ker} \vartheta_{\vec{z}, l},
$$

where $\vec{z}=\left(z_{g_{1}}(0), \ldots, z_{g_{k}}(0)\right)$ runs over all possible ordered tuples of all lengths $k \leqslant W_{1}$ consisting of representatives of level 0 such that $g_{1} \ldots g \ldots g_{k}=e$; if for a fixed tuple $\vec{z}=\left(z_{g_{1}}(0), \ldots, z_{g_{k}}(0)\right)$ of length $k$ there are several different integers $l \leqslant k+1$ such that $g_{1} \ldots g_{l-1} g g_{l} \ldots g_{k}=e$, we take the intersection over all such integers $l$. The subspaces $A_{g}(1)$ are referred to as the generalized centralizers of level 1 , elements of the $A_{g}(1)$ are called centralizers of level 1 and are denoted by $y_{g}(1)$ (under the Index Convention).

The subspace $A_{g}(1)$ has $(n, d, m)$-bounded codimension in $A_{g}$ since the intersection here is taken over an $(n, d, m)$-bounded number of subspaces of $m$-bounded codimension in $A_{g}$.

The representatives of level 1 are constructed in two different ways. First, for each $g \in G \backslash\{e\}$ we fix some elements of $A_{g}$ whose images form a basis of of the factor-space $A_{g} / A_{g}(1)$. These elements are called $b$-representatives of level 1 are denoted by $b_{g}(1) \in A_{g}$ (under the Index Convention). Since the dimensions $A_{g} / A_{g}(1)$ are $(n, d, m)$-bounded for all $g \in G \backslash\{e\}$, the total number of $b$-representatives of level 1 is $(n, d, m)$-bounded.

Second, for each pattern $\mathbf{P}=\left(*_{g} *_{g^{-1}}\right)$ of length 2 with indices $g, g^{-1} \in G \backslash\{e\}$ we consider the subspace of the factor-space $A_{e} / I_{e}$ spanned by all images of the values of $\mathbf{P}$ on homogeneous elements of $A_{g}(1), A_{g^{-1}}(1)$. Since $\operatorname{dim} A_{e} / I_{e} \leqslant m$, we can choose at most $m$ products $c=y_{g}(1) y_{g^{-1}}(1) \in A_{e}$ whose images form a basis of this subspace in $A_{e} / I_{e}$ and fix the elements $y_{g}(1), y_{g^{-1}}(1)$ involved in these representations. These elements are called $x$-representatives of level 1 and are denoted by $x_{g}(1)$ (under the Index Condition). 
Since the number of patterns under consideration is equal to $n-1$, the total number of $x$-representatives of level 1 is at most $2(n-1) m$.

By construction, if $g_{1} \ldots g_{t-1} g g_{t} \ldots g_{k}=e$, for some $t \leqslant k+1$ and $k \leqslant W_{1}$, a centralizer $y_{g}(1)$ has the following property with respect to representatives $x_{g_{j}}(0)$ of level 0 :

$$
x_{g_{1}}(0) \ldots x_{g_{t-1}}(0) y_{g}(1) x_{g_{t}}(0) \ldots x_{g_{k}}(0) \in I_{e}
$$

Definition of level $s>0$. Suppose that we have already fixed representatives of level $<s$, which are either $x$-representatives or $b$-representatives and its number is $(m, n, d)$ bounded. We now define the generalized centralizers of level s. Let $W_{s}=W_{s-1}+1=$ $2 d^{3}(n-1)+1+s$. For each $g \in G \backslash\{e\}$ we set

$$
A_{g}(s)=\bigcap_{\vec{z}} \bigcap_{l} \operatorname{Ker} \vartheta_{\vec{z}, l},
$$

where $\vec{z}=\left(z_{g_{1}}\left(\varepsilon_{1}\right), \ldots, z_{g_{k}}\left(\varepsilon_{k}\right)\right)$ runs over all possible ordered tuples of all lengths $k \leqslant W_{s}$ consisting of representatives of (possibly different) levels $<s$ (i. e., $z_{g_{u}}\left(\varepsilon_{u}\right)$ denote elements of the form $x_{g_{u}}\left(\varepsilon_{u}\right)$ or $b_{g_{u}}\left(\varepsilon_{u}\right), \varepsilon_{u}<s$, in any combination) such that

$$
g_{1} \ldots g \ldots g_{k}=e
$$

if for a fixed tuple $\vec{z}=\left(z_{g_{1}}\left(\varepsilon_{1}\right), \ldots, z_{g_{k}}\left(\varepsilon_{k}\right)\right)$ of length $k$ there are several different integers $l \leqslant k+1$ such that $g_{1} \ldots g_{l-1} g g_{l} \ldots g_{k}=e$, we take the intersection over all such integers $l$. Elements of the $A_{g}(s)$ are also called centralizers of level $s$ and are denoted by $y_{g}(s)$ (under the Index Convention).

The intersection here is taken over an $(n, d, m)$-bounded number of subspaces of $m$ bounded codimension in $A_{g}$, since the number of representatives of all levels $<s$ is $(n, d, m)$-bounded and $\operatorname{dim} A_{g} / \operatorname{Ker} \vartheta_{\vec{z}, l} \leqslant m$ for all $\vec{z}$. Hence $A_{g}(s)$ also has $(n, d, m)$ bounded codimension in the subspace $A_{g}$.

We now fix representatives of level $s$. First, for each $g \in G \backslash\{e\}$ we fix some elements of $A_{g}$ whose images form a basis of of the factor-space $A_{g} / A_{g}(s)$. These elements are denoted by $b_{g}(s) \in A_{g}$ (under the Index Convention) and are called b-representatives of level $s$. The total number of $b$-representatives of level $s$ is $(n, d, m)$-bounded, since the dimensions $A_{g} / A_{g}(s)$ are $(n, d, m)$-bounded for all $g \in G \backslash\{e\}$.

Second, for each pattern $\mathbf{P}=\left(*_{g} *_{g^{-1}}\right)$ of length 2 with indices $g \in G \backslash\{e\}$ we consider the subspace of the factor-space $A_{e} / I_{e}$ spanned by all images of values of $\mathbf{P}$ on homogeneous elements of $A_{g}(s), A_{g^{-1}}(s)$. Since $\operatorname{dim} A_{e} / I_{e} \leqslant m$, we can choose at most $m$ products $c=y_{g}(s) y_{g^{-1}}(s) \in A_{e}$ whose images form a basis of this subspace in $A_{e} / I_{e}$ and fix the elements $y_{g}(s), y_{g^{-1}}(s)$ involved in these representations. These fixed elements are called $x$-representatives of level $s$ and are denoted by $x_{g}(s)$ (under the Index Condition). The total number of $x$-representatives of level $s$ is at most $2(n-1) m$. Note that $x$-representatives of level $s$, elements $x_{g}(s)$, are also centralizers of level $s$.

It is clear from the construction that

$$
A_{g}(k+1) \leqslant A_{g}(k)
$$

for all $g \in G \backslash\{e\}$ and any $k$. 
By definition, if $g_{1} \ldots g_{t-1} g g_{t} \ldots g_{k}=e$, for some $t \leqslant k+1$ and $k \leqslant W_{s}$, then a centralizer $y_{g}(s)$ has the following property with respect to representatives of lower levels:

$$
z_{g_{1}}\left(\varepsilon_{1}\right) \ldots z_{g_{t-1}}\left(\varepsilon_{t-1}\right) y_{g}(s) z_{g_{t}}\left(\varepsilon_{t}\right) \ldots z_{g_{k}}\left(\varepsilon_{k}\right) \in I_{e}
$$

where the elements $z_{g_{j}}\left(\varepsilon_{j}\right)$ are representatives (that is, either $b_{g_{j}}\left(\varepsilon_{j}\right)$ or $x_{g_{j}}\left(\varepsilon_{j}\right)$, in any combination) of any (possible different) levels $\varepsilon_{l}<s$.

The following lemmas are direct consequences of the inclusions (2), (3) and the definitions of representatives.

Lemma 3.1. Let $g \in G \backslash\{e\}$. Then

1) every homogeneous element $a_{e} \in A_{e}$ can be represented modulo $I_{e}$ as a linear combination of representatives $x_{e}(0)$ of level 0 .

2) every product $a_{g} b_{g^{-1}}$ in homogeneous elements can be represented modulo $I_{e}$ as a linear combination of products of the same pattern in representatives of level 0.

$3)$ every product $y_{g}\left(k_{1}\right) y_{g^{-1}}\left(k_{2}\right)$ in centralizers of levels $k_{1}, k_{2}$ can be represented modulo $I_{e}$ as a linear combination of products $x_{g}(s) x_{g^{-1}}(s)$ of the same pattern in $x$-representatives of any level $s$ satisfying $0 \leqslant s \leqslant \min \left\{k_{1}, k_{2}\right\}$.

Lemma 3.2. Let $y_{g}(l+1)$ be a centralizer of level $l+1, b_{h}(l)$ be $b$-representative of level $l$ with $g, h, g h \in G \backslash e$. Then elements of the form $u_{g h}=y_{g}(l+1) b_{h}(l)$ or $v_{h g}=b_{h}(l) y_{g}(l+1)$ are centralizers of level $l$.

Proof. The proof follows directly from (3) and the definitions of $W_{i}$.

Lemma 3.3. Any product of the form $a_{g^{-1}} y_{g}(k+1)$ or $y_{g}(k+1) a_{g^{-1}}$, where $y_{g}(k+1)$ is a centralizer of level $k>0$, is equal modulo $I_{e}$ to a product of the form $y_{g^{-1}}(k) y_{g}(k)$ or accordingly $y_{g}(k) y_{g^{-1}}(k)$, where $y_{g^{-1}}(k), y_{g}(k)$ are centralizers of level $k-1$.

Proof. We represent $a_{g^{-1}}$ as a sum of a centralizer $y_{g^{-1}}(k)$ of level $k$ and a linear combination of $b$-representatives $b_{g^{-1}}(k)$ of level $k$ and substitute this sum into the product $a_{g^{-1}} y_{g}(k+1)$. We obtain a sum of the element $y_{g^{-1}}(k) y_{g}(k+1)$ and a linear combination of elements of the form $b_{g^{-1}}(k) y_{g}(k+1)$. By (3) the product $b_{g^{-1}}(k) y_{g}(k+1)$ belongs to $I_{e}$. Hence $a_{g^{-1}} y_{g}(k+1)=y_{g^{-1}}(k) y_{g}(k+1)\left(\bmod I_{e}\right)$. Similarly, $y_{g^{-1}}(k+1) a_{g}=$ $y_{g^{-1}}(k+1) y_{g}(k)\left(\bmod I_{e}\right)$. Since $A_{g}(k) \geqslant A_{g}(k+1)$, both products have the required form.

Construction of nilpotent ideal. Recall that $N=d^{2}+3$ is the fixed notation for the highest level. We have constructed the generalized centralizers $A_{g}(N)$ for $g \in G \backslash\{e\}$. Let $G \backslash\{e\}=\left\{g_{1}, \ldots, g_{n-1}\right\}$ We set

$$
Z={ }_{\mathrm{id}}\left\langle A_{g_{1}}(N), A_{g_{2}}(N), \ldots, A_{g_{n-1}}(N), I_{e}\right\rangle .
$$

This ideal has $(n, d, m)$-bounded codimension in $A$, since each subspace $A_{h}(N), h \in$ $G \backslash\{e\}$, has $(n, d, m)$-bounded codimension in $A_{h}$, while the dimension of $A_{e} / I_{e}$ is at most $m$ by hypothesis. To prove the Theorem 1.2 we show that the ideal $Z$ is nilpotent of $(n, d)$-bounded class.

Definition. For every $g \in G$ we set $Z_{g}=Z \cap A_{g}$. 
Lemma 3.4. The subspace $Z_{e}$ is contained modulo $I_{e}$ in the subspace spanned by products of the form $y_{h^{-1}}(N-2) y_{h}(N-2)$ and by products of the form $a_{g^{-1}} i_{e} a_{g}$, where $y_{h^{-1}}(N-2)$, $y_{h}(N-2)$ are centralizers of level $N-2, a_{g^{-1}} \in A_{g^{-1}}, a_{g} \in A_{g}, i_{e} \in I_{e}, h, g \in G \backslash\{e\}$.

Proof. An element of $Z_{e}$ is modulo $I_{e}$ a linear combination of products of the forms:

$$
\begin{gathered}
a_{g^{-1}} i_{e} a_{g}, \text { where } a_{g^{-1}} \in A_{g}, i_{e} \in I_{e}, a_{g} \in A_{g}, g \neq e\{e\} \\
a_{g^{-1}} y_{g}(N) \text {, where } a_{g^{-1}} \in A_{g^{-1}}, g \neq e, y_{g}(N) \in A_{g}(N), \\
y_{g}(N) a_{g^{-1}} \text {, where } g \neq e, y_{g}(N) \in A_{g}(N), a_{g^{-1}} \in A_{g^{-1}}, \\
a_{g_{1}} y_{g}(N) a_{g_{2}}, \text { where } a_{g_{1}} \in A_{g_{1}}, a_{g_{2}} \in A_{g_{2}}, y_{g}(N) \in A_{g}(N), g_{1} g g_{2}=e,
\end{gathered}
$$

The product (4) is already of the required form. By Lemma 3.3 the products $y_{g}(N) a_{g^{-1}}$ and $a_{g^{-1}} y_{g}(N)$ can be represented modulo $I_{e}$ as linear combinations of products of the form $y_{g}(N-1) y_{g^{-1}}(N-1)$ and therefore have also the required representation since $A_{g}(N-1) \leqslant A_{g}(N-2)$.

Consider the product (7). Since $g_{1} g g_{2}=e$ and $g \neq e$, at least one $g_{i}, i=1,2$ is not equal to $e$. Let, for example, $g_{1} \neq e$. We represent $a_{g_{1}}$ as a sum of a centralizer $y_{g^{-1}}(N-1)$ of level $N-1$ and a linear combination of $b$-representatives $b_{g^{-1}}(N-1)$ of level $N-1$ and insert this expression into (7). We obtain a linear combination of products of the following two forms

$$
y_{g_{1}}(N-1) y_{g}(N) a_{g_{2}}
$$

and

$$
b_{g_{1}}(N-1) y_{g}(N) a_{g_{2}} .
$$

In (8) we set $a_{g_{1}^{-1}}:=y_{g}(N) a_{g_{2}}$. Applying Lemma 3.3 and the inclusions (2) to $y_{g_{1}}(N-$ 1) $a_{g_{1}^{-1}}$ we obtain that (8) is equal modulo $I_{e}$ to a product of the required form $y_{g_{1}}(N-$ 2) $y_{g_{1}^{-1}}(N-2)$.

Let us now consider the product (9). If $g_{2}=e$, then $g_{1} g=e$ and $b_{g_{1}}(N-1) y_{g}(N) \in I_{e}$ by (3). Since $I_{e}$ is an ideal of $A_{e}$ and $g_{2}=e$,

$$
b_{g_{1}}(N-1) y_{g}(N) a_{e} \in I_{e} .
$$

If $g_{2} \neq e$, then $g_{1} g \neq e$ and $b_{g_{1}}(N-1) y_{g}(N)$ is a a centralizer of level $N-1$ by Lemma 3.2.

$$
b_{g_{1}}(N-1) y_{g}(N) a_{g_{2}}=y_{g_{1} g}(N-1) a_{g_{2}} .
$$

Again by Lemma 3.3 the product $y_{g_{1} g}(N-1) a_{g_{2}}$ is equal modulo $I_{e}$ to the product of the require form $y_{g_{1} g}(N-2) y_{g_{2}}(N-2)$. The case where $g_{1}=e, g_{2} \neq e$ in (7) can be treated in the same manner.

Proof of Theorem 1.2. We set $H=d^{2}+1, T=d(H-1)+1=d^{3}+1, \quad S=$ $(T-1)(n-1)+1=d^{3}(n-1)+1, \quad U=d(n-1)$, and $Q=(U+1)(S-1)+1=$ $(d(n-1)+1) d^{3}(n-1)=d^{4}(n-1)^{2}+d^{3}(n-1)$. By Lemma 2.2 it suffices to show that $\left(Z_{e}\right)^{Q}=0$. 
We consider an arbitrary product of length $Q$ in elements $c_{i}$ from $Z_{e}$ :

$$
c_{1} c_{2} \ldots c_{Q}
$$

(here the indices are numbering). By Lemma 3.4 we can represent modulo $I_{e}$ every $c_{k}$ as a linear combination of products of some special form. Substituting these expressions into (10) we obtain a linear combination of elements

$$
z_{1} z_{2} \ldots z_{Q}
$$

where the $z_{k}$ (here the indices are also numbering) are either elements $i_{e} \in I_{e}$ or products $c_{e}=a_{g^{-1}} w_{e} a_{g} \in A_{e}, w_{e} \in I_{e}$ or products $v_{e}=y_{g_{k}^{-1}}(N-2) y_{g_{k}}(N-2) \in A_{e}$, in centralizers $y_{g_{k}^{-1}}(N-2), y_{g_{k}}(N-2)$ of level $N-2$.

If in (11) among $z_{k}$ there are at least $d$ occurrences of elements $i_{e} \in I_{e}$ the summand is trivial, since $I_{e}$ is an ideal of $A_{e}$ and $\left(I_{e}\right)^{d}=0$.

Suppose now that in (11) there are at least $(d-1) n+1$ entries of products $c_{e}=$ $a_{g^{-1}} i_{e} a_{g}$. Among them we can choose $d$ products $c_{e}=a_{g_{k}^{-1}} i_{e} a_{g_{k}} \in A_{e}$ with the same pair of indices $g_{k}^{-1}, g_{k}$ :

$$
z_{1} \ldots z_{l_{1}} \underbrace{a_{g_{k}^{-1}} i_{e} a_{g_{k}}} z_{l_{1}+1} \ldots z_{l_{2}} \underbrace{a_{g_{k}^{-1}} i_{e} a_{g_{k}}} z_{l_{2}} \ldots z_{l_{k}} \underbrace{a_{g_{k}^{-1}} i_{e} a_{g_{k}}} z_{l_{k}+1} \ldots z_{Q} .
$$

Since the products $a_{g_{k}} z_{l_{s}+1} \ldots z_{l_{s+1}} a_{g_{k}^{-1}}$ between the elements $i_{e}$ belong to $A_{e}, I_{e}$ is an ideal in $A_{e}$ and $\left(I_{e}\right)^{d}=0$, then the product (11) is equal to 0 .

Consider the case where the number of $i_{e}$-occurrences in (11) is at most $d$ and the number of $c_{e}$-occurrences is at most $U=d(n-1)$. Since $Q=(U+d+1)(S-1)+1$, the product (11) has at least one subproduct consisting of $S$ elements $v_{e}$ (going one after another):

$$
\left(y_{g_{1}^{-1}}(N-2) y_{g_{1}}(N-2)\right)\left(y_{g_{2}^{-1}}(N-2) y_{g_{2}}(N-2)\right) \ldots\left(y_{g_{S}^{-1}}(N-2) y_{g_{S}}(N-2)\right),
$$

where $y_{g_{i}}(N-2) \in A_{g_{i}}(N-2), y_{g_{i}^{-1}}(N-2) \in A_{g_{i}^{-1}}(N-2)$ are (possibly different) centralizers of level $N-2$. Since $S=(T-1)(n-1)+1$ in (12) there are at least $T$ entries of products $y_{g_{i}^{-1}}(N-2) y_{g_{i}}(N-2)$ with the same pair of indices, say, $g_{k}^{-1}, g_{k}$. We choose any $T$ such products and represent modulo $I_{e}$ all the other pairs as linear combinations of products of representatives of level 0 by Lemma 3.1;

$$
w_{e} \ldots w_{e}\left(y_{g_{k}^{-1}}(N-2) y_{g_{k}}(N-2)\right) w_{e} \ldots w_{e}\left(y_{g_{k}^{-1}}(N-2) y_{g_{k}}(N-2)\right) \ldots,
$$

where there are $T$ occurrences of (possibly different) products $y_{g_{k}^{-1}}(N-2), y_{g_{k}}(N-2)$ with the same pair of indices $g_{k}^{-1}, g_{k}$, the $w_{e}$ are possibly different elements of $A_{e}$ : either $i_{e} \in I_{e}$ or representatives $x_{e}(0)$. If in (13) among $w_{e}$ there are at least $d$ occurrences of elements of $I_{e}$ the summand is trivial, since $I_{e}$ is an ideal of $A_{e}$ and $\left(I_{e}\right)^{d}=0$. In the opposite case, as $T=d(H-1)+1$, there is a subproduct of the form

$$
\left(y_{g_{k}^{-1}}(N-2) y_{g_{k}}(N-2)\right) x_{e}(0) \ldots x_{e}(0)\left(y_{g_{k}^{-1}}(N-2) y_{g_{k}}(N-2)\right) \ldots,
$$


where there are $H=d^{2}+1$ occurrences of products $y_{g_{k}^{-1}}(N-2) y_{g_{k}}(N-2)$ and between them there are only $x$-representative of level 0 and no elements from $I_{e}$. By lemma 3.1 we represent modulo $I_{e}$ the first entry $y_{g_{k}^{-1}}(N-2) y_{g_{k}}(N-2)$ as a linear combination of the products of the same pattern in representatives in level 1 , the second - in level 2 , and so on, the last one - in level $H$. We obtain a linear combination

$$
\left(x_{g_{k}^{-1}}(1) x_{g_{k}}(1)+i_{e}\right) x_{e}(0) \ldots x_{e}(0)\left(x_{g_{k}^{-1}}(2) x_{g_{k}}(2)+i_{e}\right) \ldots\left(x_{g_{k}^{-1}}(H) x_{g_{k}}(H)+i_{e}\right) .
$$

Expanding this expression we get a linear combination of products of the form

$$
c_{1} x_{e}(0) \ldots x_{e}(0) c_{2} x_{e}(0) \ldots x_{e}(0) \ldots c_{H},
$$

(here the indices are numbering) where the $c_{k}$ are either elements $i_{e} \in I_{e}$ or products $x_{g_{k}^{-1}}\left(n_{k}\right) x_{g_{k}}\left(n_{k}\right)$ of different levels with one and the same pair of indices $g_{k}^{-1}, g_{k} \in G$. If in a summand there are at least $d$ entries of $i_{e} \in I_{e}$ it is trivial by assumptions. In the summands with less than $d$ entries of the $i_{e}$, we can find an interval long enough without $i_{e}$-entries. More precisely, since $H=d^{2}+1$ there is a subproduct of the form

$$
\left(x_{g_{k}^{-1}}(s) x_{g_{k}}(s)\right) x_{e}(0) \ldots x_{e}(0)\left(x_{g_{k}^{-1}}(s+1) x_{g_{k}}(s+1)\right) \ldots\left(x_{g_{k}^{-1}}(s+d) x_{g_{k}}(s+d)\right),
$$

where there are $d+1$ products $y_{g_{k}^{-1}}(l) y_{g_{k}}(l)$ of different levels $l=s, \ldots, s+d$. For each $t=0, \ldots, d-1$, the product

$$
x_{g_{k}}(s+t) x_{e}(0) \ldots x_{e}(0) x_{g_{k}^{-1}}(s+t+1)
$$

includes exactly one centralizer of level $s+t+1$, all the other elements are representatives of lower levels, and the weight of the product is at most $2 S=2 d^{3}(n-1)+2=W_{1} \leqslant W_{s+t+1}$. By (3)

$$
x_{g_{k}}(s+t) x_{e}(0) \ldots x_{e}(0) x_{g_{k}^{-1}}(s+t+1) \in I_{e}
$$

for all $t=0, \ldots, d-1$. It follows that (??) is equal to product

$$
x_{g_{k}^{-1}}(s) \underbrace{\left(i_{e} i_{e} \ldots i_{e}\right)}_{d} x_{g_{k}}(s+d)=0
$$

which is trivial, since $\left(I_{e}\right)^{d}=0$.

\section{Proof of the main result}

In this section we prove Theorem 1.1. Recall that we are given an associative algebra $A$ over a field $F$ that admits a finite soluble automorphism group $G$ of order $n$ prime to the characteristic of $F$ such that the fixed-point subalgebra $A^{G}$ has a two-sided nilpotent ideal $I \triangleleft A^{G}$ of nilpotency index $d$ and of finite codimension $m$ in $A^{G}$. The aim is to find a nilpotent ideal in $A$ of $(n, d)$-bounded nilpotency index and of finite $(n, d, m)$-bounded codimension.

Proof of Theorem 1.1. First, we consider the case where $G$ is a cyclic group of prime order $p$. Let $g$ be a generator of $G$. Then $g$ induces an automorphism of the algebra 
$A \otimes_{\mathbb{Z}} \mathbb{Z}[\omega]$, where $\omega$ is a primitive $p$-th root of unity. The fixed-point subalgebra of this automorphism denoted by the same letter has the same dimension $m$ over the field extended by $\omega$. It suffices to prove Theorem 1.1 for the algebra $A \otimes_{\mathbb{Z}} \mathbb{Z}[\omega]$. Hence in what follows we can assume that the ground field $F$ contains $\omega$. We define the homogeneous components $A_{k}$ for $k=0, \ldots, p-1$ as the subspaces

$$
A_{k}=\left\{a \in A \mid a^{g}=\omega^{k} a\right\} .
$$

Since the characteristic of $F$ does not divide $p$, we have

$$
A=A_{0} \oplus A_{1} \oplus \cdots \oplus A_{p-1} .
$$

This decomposition determines a grading on $A$ by a cyclic group of prime order $p$, with $A_{0}=A^{G}$ in view of the obvious inclusions

$$
A_{s} A_{t} \subseteq A_{s+t},
$$

where $s+t$ is computed modulo $p$. Hence the case $|G|=p$ in Theorem 1.1 follows from Theorem 1.2.

Let now $G$ be any finite soluble group of automorphisms of $A$, and suppose that its order $n$ is not divisible by the characteristic of $F$. We use induction on $|G|$. We may assume that $n$ is not a prime number. This means that in $G$ there is a non-trivial normal subgroup $H$. We consider the subalgebra $C=A^{H}$ of its fixed points. Since $H \triangleleft G$, we have $C^{g} \leqslant C$ for any $g \in G$. The subalgebra $C$ admits a finite solvable group of automorphisms of order $\leqslant|G / H|$ which is strictly less than $|G|$ and not divisible by the characteristic of $F$. By induction $C$ has a nilpotent ideal $J \triangleleft C$ of $(|G / H|, d, m)$ bounded codimension $t=t(|G / H|, d, m)$ and of $(|G / H|, d)$-bounded nilpotency index $h=h(|G / H|, d)$. By Lemma 2.3 there exists a nilpotent $G$-invariant ideal $K \geqslant J$ in $A$ of nilpotency index $h_{1}=h_{1}(|H|, h)$ bounded by $|H|$ and by the nilpotency index of $J$. The subgroup $H$ acts on the factor-algebra $\bar{A}=A / K$ and subalgebra of fixed points $\bar{A}^{H}$ has dimension at most $t$. We apply induction hypothesis to the algebra $\bar{A}$ and the automorphism group $H$ of Aut $\overline{\mathrm{A}}$ whose order is strictly less than $|G|$. The algebra $\bar{A}$ has a nilpotent ideal $Z$ of $(|H|, t)$-bounded codimension and of $|H|$-bounded nilpotent index $h_{2}=h_{2}(|H|)$. The image of $Z$ in $A$ is a required ideal since its nilpotency index is at most $h_{1} h_{2}$, which is a $(n, d)$-bounded number, and the codimension is $(n, d, m)$-bounded.

\section{References}

[1] G. M. Bergman, I. M. Isaacs, Rings with fixed-point-free group actions, Proc. London. Math. Soc. 27, N 3 (1973), 69-87.

[2] V.K. Kharchenko, Automorphisms and Derivations of Associative Rings, KLUWER ACADEMIC PUBLISHERS, 1991.

[3] E. I. Khukhro, Groups and Lie rings admitting an almost regular automorphism of prime order, Math. USSR Sb. 71 (1992) 51-63. 
[4] E. I. Khukhro, N. Yu. Makarenko, Lie rings with almost regular automorphisms, J. Algebra, 264, N 2 (2003), 641-664.

[5] N. Yu. Makarenko, E. I. Khukhro, Almost solubility of Lie algebras with almost regular automorphisms, J. Algebra, 277, N 1 (2004), 370-407.

[6] N. Yu. Makarenko, Lie type algebras with an automorphism of finite order, $J$. Algebra, 439 (2015), 33-66. 